\title{
Leptomeningeal Myelomatosis: A Rare but Devastating Manifestation of Multiple Myeloma Diagnosed Using Cytology, Flow Cytometry, and Fluorescent in situ Hybridization
}

\author{
Martin Bommer ${ }^{\mathrm{a}}$ Miriam Kull $^{\mathrm{b}}$ Veronica Teleanu ${ }^{\mathrm{b}}$ Phyllis Schwarzwälder ${ }^{\mathrm{b}}$ \\ Manuela Feuring-Buske ${ }^{b}$ Jan Kroenke ${ }^{b}$ Donald Bunjes ${ }^{b}$ Christian Langer $^{b}$ \\ ${ }^{a}$ Hematology, Oncology, Palliative Care and Infectious Diseases, Department of Internal Medicine, Alb-Fils-Kliniken, \\ Göppingen, Germany; ${ }^{b}$ Hematology, Oncology, Palliative Care, Rheumatology and Infectious Diseases, Department \\ of Internal Medicine III, University Hospital of Ulm, Ulm, Germany
}

\section{Keywords \\ Leptomeningeal myelomatosis · Neoplastic meningitis . Cytology · Cerebrospinal fluid · Multiple myeloma · Flow cytometry $\cdot$ Fluorescence in situ hybridization}

\begin{abstract}
Introduction: Involvement of the central nervous system in patients with multiple myeloma is a rare event. We evaluated the diagnostic workup and prognosis of patients with leptomeningeal myelomatosis (LMM). Methods: Between April 2005 and April 2016, we identified 16 cases with LMM. The involvement was diagnosed by magnetic resonance imaging (MRI), cerebrospinal fluid (CSF) cytology as well as flow cytometry. Fluorescence in situ hybridization (FISH) was used in 8/16 cases. In 1 case, genome-wide screening for genetic alterations using single-nucleotide polymorphism (SNP) array analysis was performed. Results: The median time from initial diagnosis until the occurrence of LMM was 434 days. At diagnosis, the median age was 60 years. The median cell count was $21 / \mu \mathrm{L}$ (range $1-1,333 / \mu \mathrm{L}$ ). All CSF samples showed malignant pleocytosis, confirmed by flow cytometry in $12 / 16$ patients. FISH revealed high-risk features
\end{abstract}

\section{KARGER}

(c) 2018 S. Karger AG, Basel

E-Mail karger@karger.com

www.karger.com/aha in the majority of samples. Treatment for LMM consisted of intrathecal chemotherapy and radiation therapy. Genomewide screening assays revealed different subclones. The outcome was dismal with a median overall survival after the diagnosis of LMM of 82 days. Conclusion: By combining several technical procedures, it is possible to identify most patients with LMM. Management of affected patients is challenging and the survival short after a diagnosis of LMM.

(c) 2018 S. Karger AG, Basel

\section{Introduction}

In contrast to other malignancies, the frequency of leptomeningeal myelomatosis (LMM) is as low as $1 \%$ [1]. Only a few comprehensive overviews with $>10$ patients have been published [1-7]. LMM is defined as the presence of clonal plasma cells in the cerebrospinal fluid

Martin Bommer, MD

Hematology, Oncology, Palliative Care and Infectious Diseases

Department of Internal Medicine, Alb-Fils Kliniken

Am Eichert 3, DE-73035 Göppingen (Germany)

E-Mail martin.bommer@af-k.de

Christian Langer, MD

Department of Internal Medicine III, University Hospital Ulm

Albert-Einstein-Allee 23

DE-89081 Ulm (Germany)

E-Mail christian.langer@uni-ulm.de 


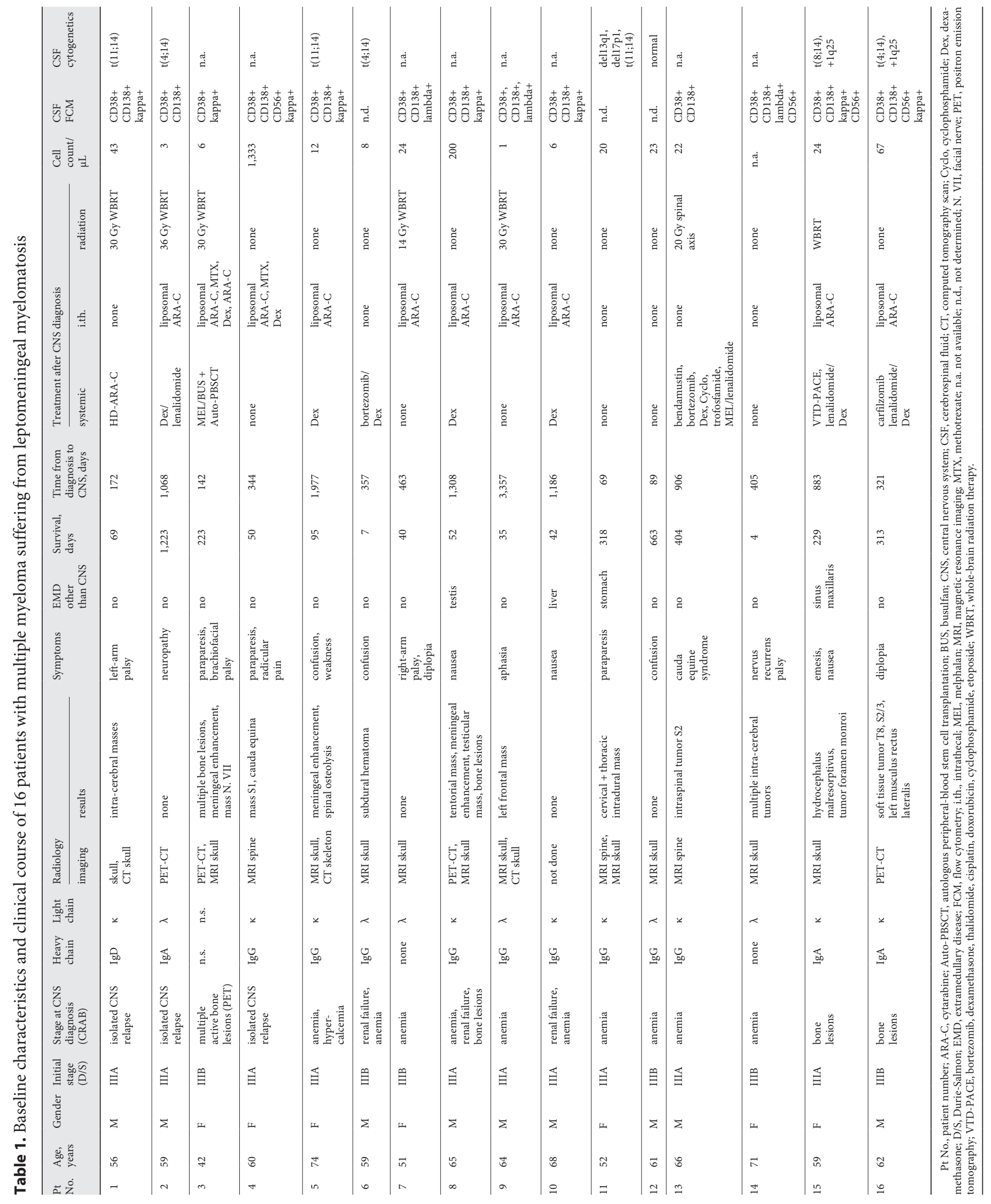


Fig. 1. Flow cytometry of cerebrospinal fluid from patient No. 3. Plasma cells were gated according to standard protocols and showed light chain $\kappa$ expression.

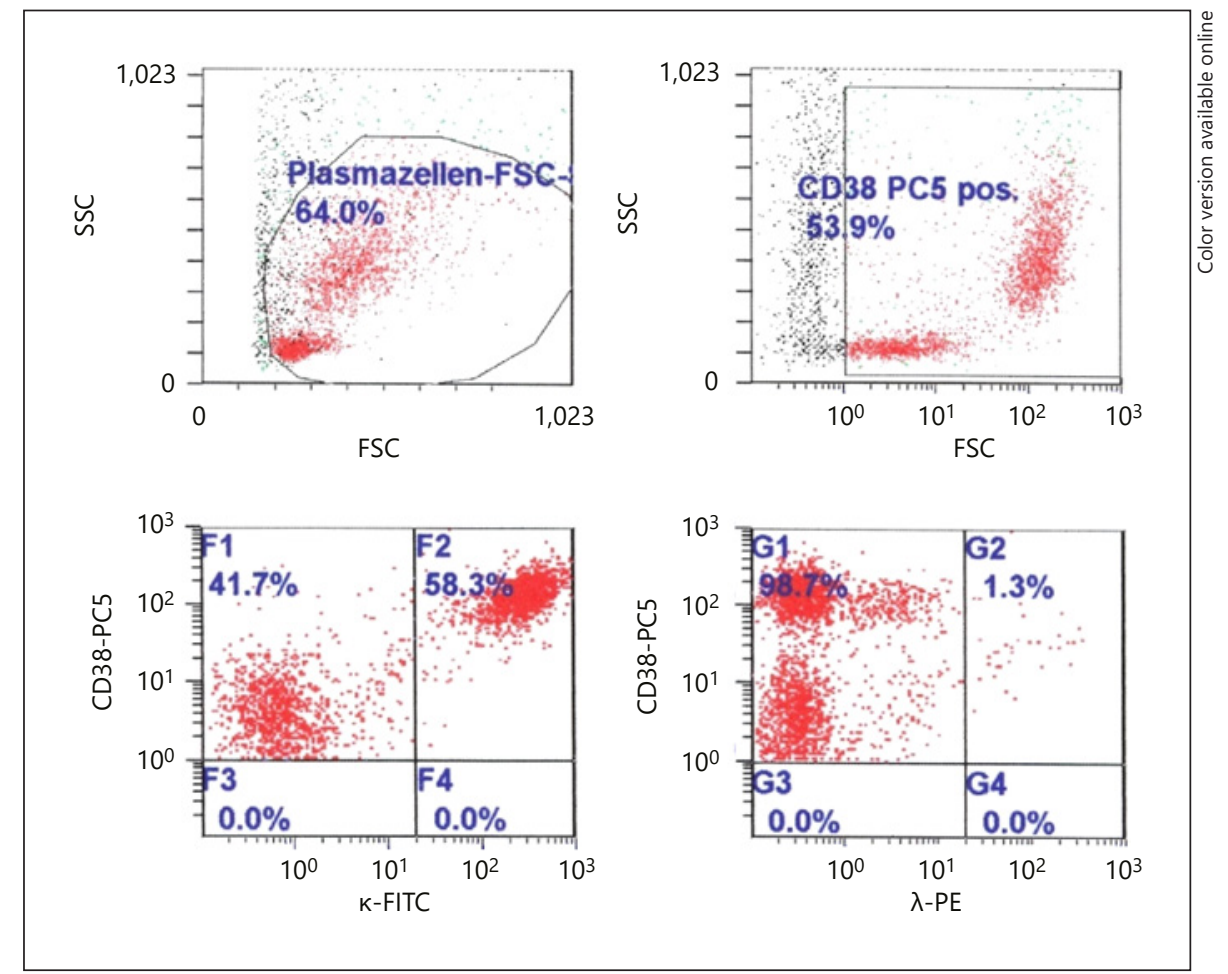

(CSF). To establish a diagnosis of LMM, either a typical leptomeningeal enhancement on magnetic resonance imaging (MRI) and/or evidence of clonal plasma cells in the CSF is necessary. CSF cytology in malignancies other than myeloma has a limited sensitivity of around $50-60 \%$, but excellent specificity (>95\%) when done by an experienced cytologist [8]. Fluorescence-activated cell sorting (FACS) can increase the sensitivity of the detection of tumor cells in patients with aggressive lymphoma at a high risk of central nervous system (CNS) involvement $[9,10]$, and patients with atypical or suspicious CSF morphology. Therefore, adding flow cytometry may also improve the diagnostic sensitivity and specificity in patients with multiple myeloma (MM). Risk factors for leptomeningeal disease in MM are plasmoblastic morphology, extramedullary disease, elevated lactate dehydrogenase (LDH) and high-risk cytogenetics such as the presence of a translocation $\mathrm{t}(4 ; 14)$ and/or a deletion (del) of the chromosomal band 13q14 [2,3].

\section{Materials and Methods}

\section{Patients}

Between 2004 and 2016, we identified 16 myeloma patients with leptomeningeal disease treated at the University Hospital of
Ulm in Germany. All of them were suffering from symptomatic MM and CSF analysis was triggered by CNS-related symptoms. CSF samples for cytology, FACS, and cytogenetic studies were drawn by lumbar puncture during standard clinical workup. All patients gave their written informed consent for the procedure.

This retrospective study was conducted in accordance with the principles of the Helsinki Declaration. The local ethics committee approved the evaluation protocol.

\section{CSF Analysis}

CSF was obtained by lumbar puncture. Precautions were taken to minimize contamination by peripheral blood.

The fresh specimens were processed following standard procedures as previously published $[11,12]$. All smears were analyzed by M.B. Additional FACS analysis was done within a few hours of sampling using standard procedures. CSF volume ranged from 1.5 to $6 \mathrm{~mL}$. The results were analyzed by M.F.-B. and M.B. Samples were judged to be malignant only if a distinct cell population with the disease specific immune-phenotype, i.e., CD38+, CD138+, or a light chain restriction, could be detected.

Fluorescence in situ hybridization (FISH) in combination with immunofluorescent detection of light chain-restricted plasma cells (cIg-FISH) was performed according to standardized protocols using commercially available probes (Abbott Molecular; MetaSystems).

Single-nucleotide polymorphism (SNP) chip analysis was performed from total genomic DNA (500 ng), digested and ligated to adaptors. A generic primer that recognizes the adaptor sequence was used to amplify adaptor-ligated DNA fragments. Then, the amplified DNA was fragmented, labeled, and hybridized to the hu- 


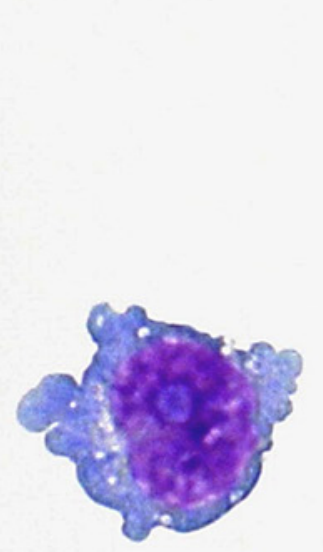

a
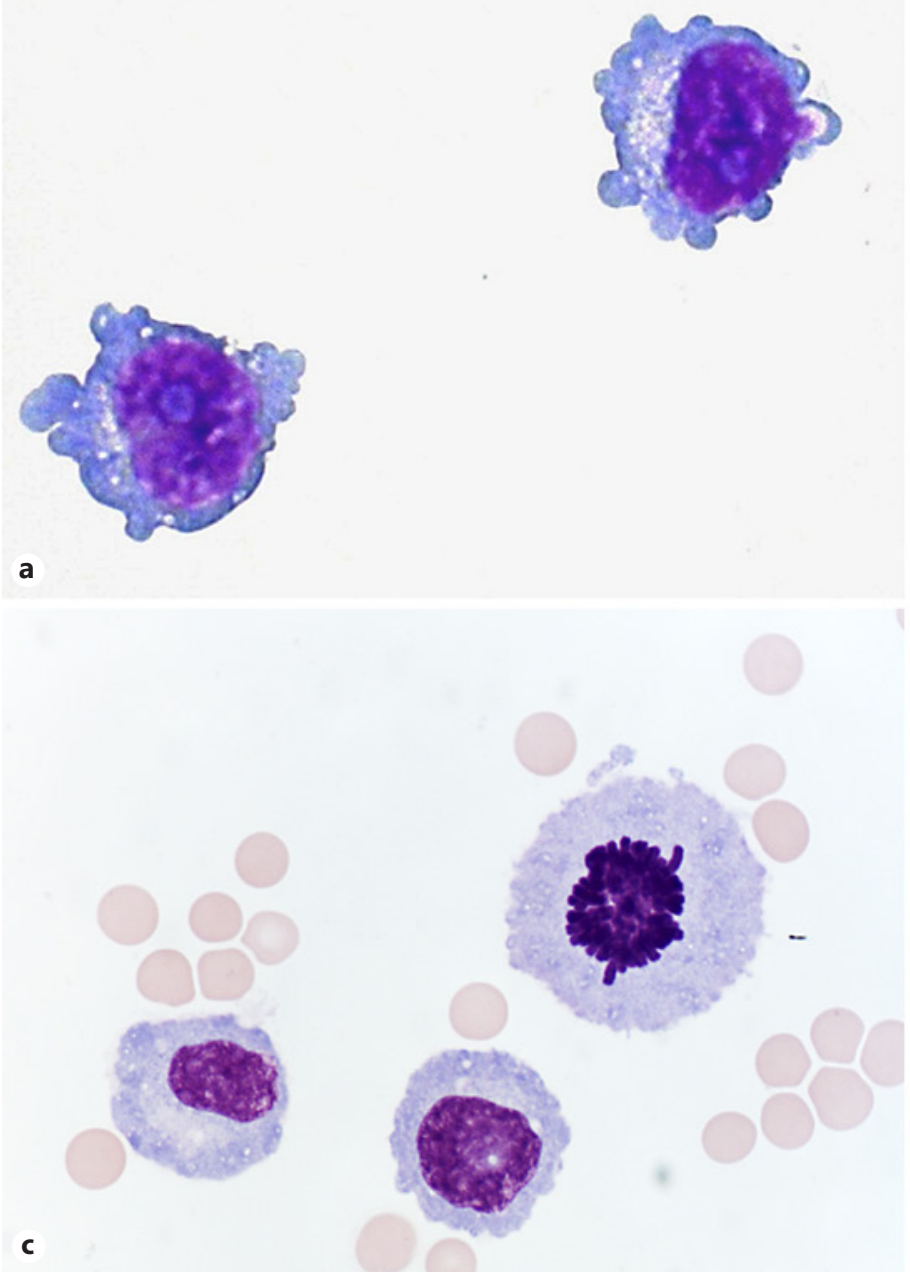

b
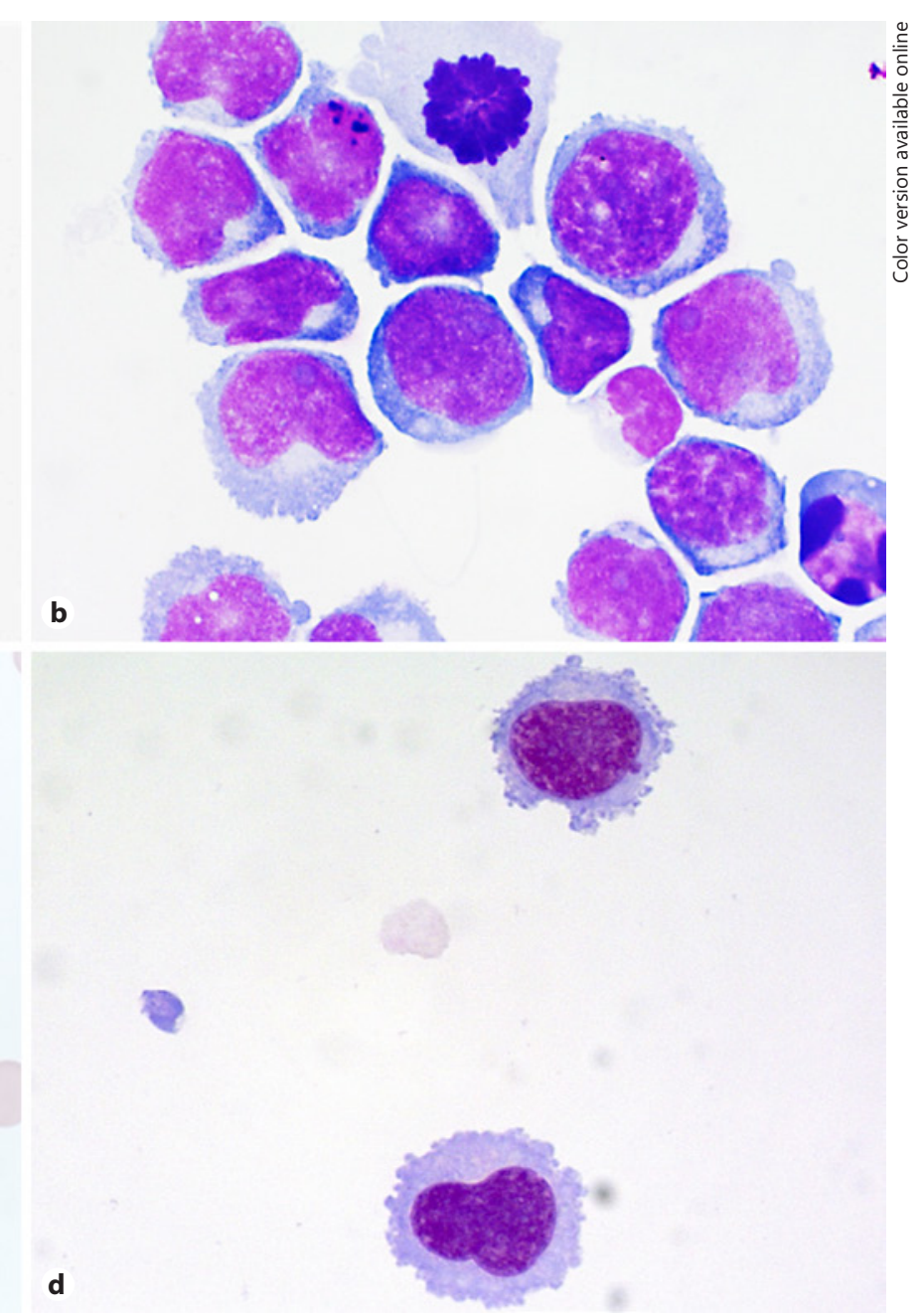

d

Fig. 2. a-d Cytomorphology of 4 representative cases of patients with multiple myeloma affected by leptomeningeal myelomatosis.

man SNP array 6.0 (Affymetrix) for $16 \mathrm{~h}$, followed by automated washing, staining, and scanning. Data analysis was done with a standard software Affymetrix genotyping console ${ }^{\mathrm{TM}}$.

For statistical analysis, GraphPad Prism (La Jolla, CA, USA) software was used.

\section{Results}

All patients had symptomatic disease with very variable neurologic symptoms. Most of them suffered from central nerve palsy, pain, or changes of mental state. Radiographic studies (MRI and CT scan) revealed pathology in 12 of 16 patients, but only 2 showed meningeal enhancement. The median cell count was $21 / \mu \mathrm{L}$ with a wide range $(1-1,333 / \mu \mathrm{L})$. FACS could be performed in 13 out of 16 patients. All samples showed clonal disease with the expression of CD38 and also CD138 or $\kappa / \lambda$ light chain restriction. In 4 samples (patient Nos. 4, 14, 15, and 16) additional expression of CD56 was found (Table 1). Due to low cell counts in some patients (Nos. 2, 3, 9, and 10), FACS added important information for the interpretation of CSF cytology (Fig. 1). In 1 case (patient No. 6), FACS could not be performed because of the small amount of CSF available for analysis, but cytomorphology was unambiguous (Fig. 2).

The median time from a diagnosis of MM to the diagnosis of leptomeningeal spread was 434 days (range 693,357 days). All patients finally died (range 7-1,233 days after the diagnosis of LMM). The majority of patients received a specific treatment, except for those with a clearly dismal prognosis, i.e., a life expectancy of less than a few days (i.e., patient No. 6). The median survival time was 82 
Fig. 3. Overall survival of 16 patients with multiple myeloma suffering from leptomeningeal myelomatosis (LMM) with a median survival of 82 days after the diagnosis of LMM.

Fig. 4. Characterization of tumor material from patient No. 15. At the time of leptomeningeal myelomatosis presentation, tumor material from different locations was available for analysis. a Copy number alterations of chromosome 8. Plasma cells from the CSF present with a gain of $8 \mathrm{q}$ and a small biallelic gain. In contrast, the plasma cells in the BM present with a loss of $8 \mathrm{p}$. b cIg-FISH of plasma cells from CSF demonstrating a $\mathrm{t}(8 ; 14) . \mathrm{GC}$, germline control; CSF, cerebrospinal fluid; BM, bone marrow.
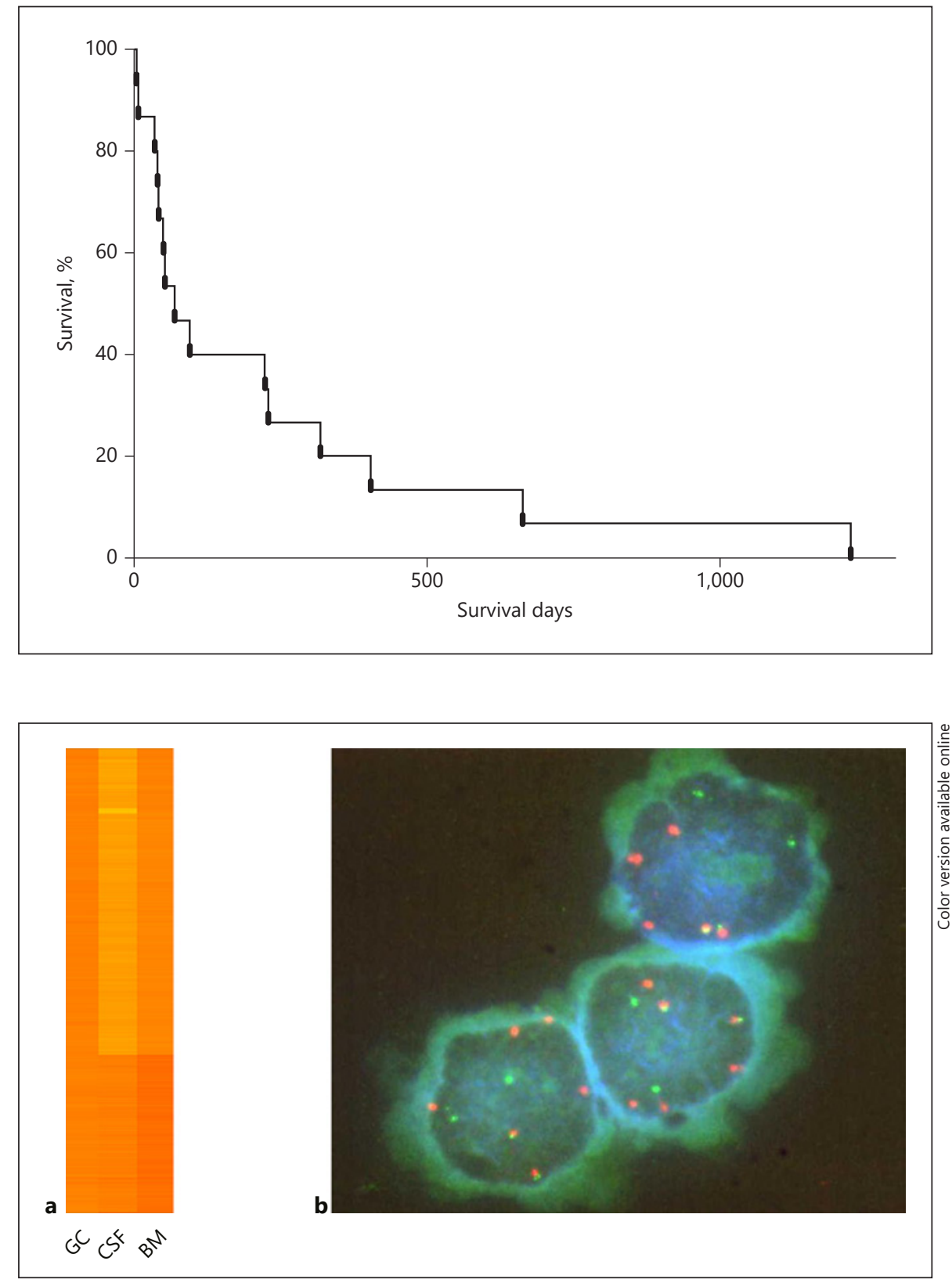

days; the Kaplan-Maier estimated survival is presented in Figure 3. An overview of the clinical data and laboratory tests is given in Table 1.

In 1 patient, a more detailed molecular characterization of the disease was possible. In contrast to the presenting bone marrow (BM) 18 months earlier, at the time of progression, a translocation $\mathrm{t}(8 ; 14)$ was detectable in the CSF as well as in the BM (Fig. 4); despite the shared acquired IgH translocation, copy number alteration analysis revealed different subclones at these 2 clinical sites.

\section{Discussion}

The diagnosis of LMM is based upon MRI and cytological examination of the CSF. MRI is of limited use in leukemia and lymphoma, as previously described [13]; in patients with multiple myeloma, however, MRI adds important diagnostic information. In a large case study published by Paludo et al. [6], only $17 \%$ of the patients had no pathologic finding on MRI. Most of them had meningeal enhancement (70\%) consistent with LMM, and MRI also 
Fig. 5. Proposed algorithm for the identification of leptomeningeal myelomatosis.

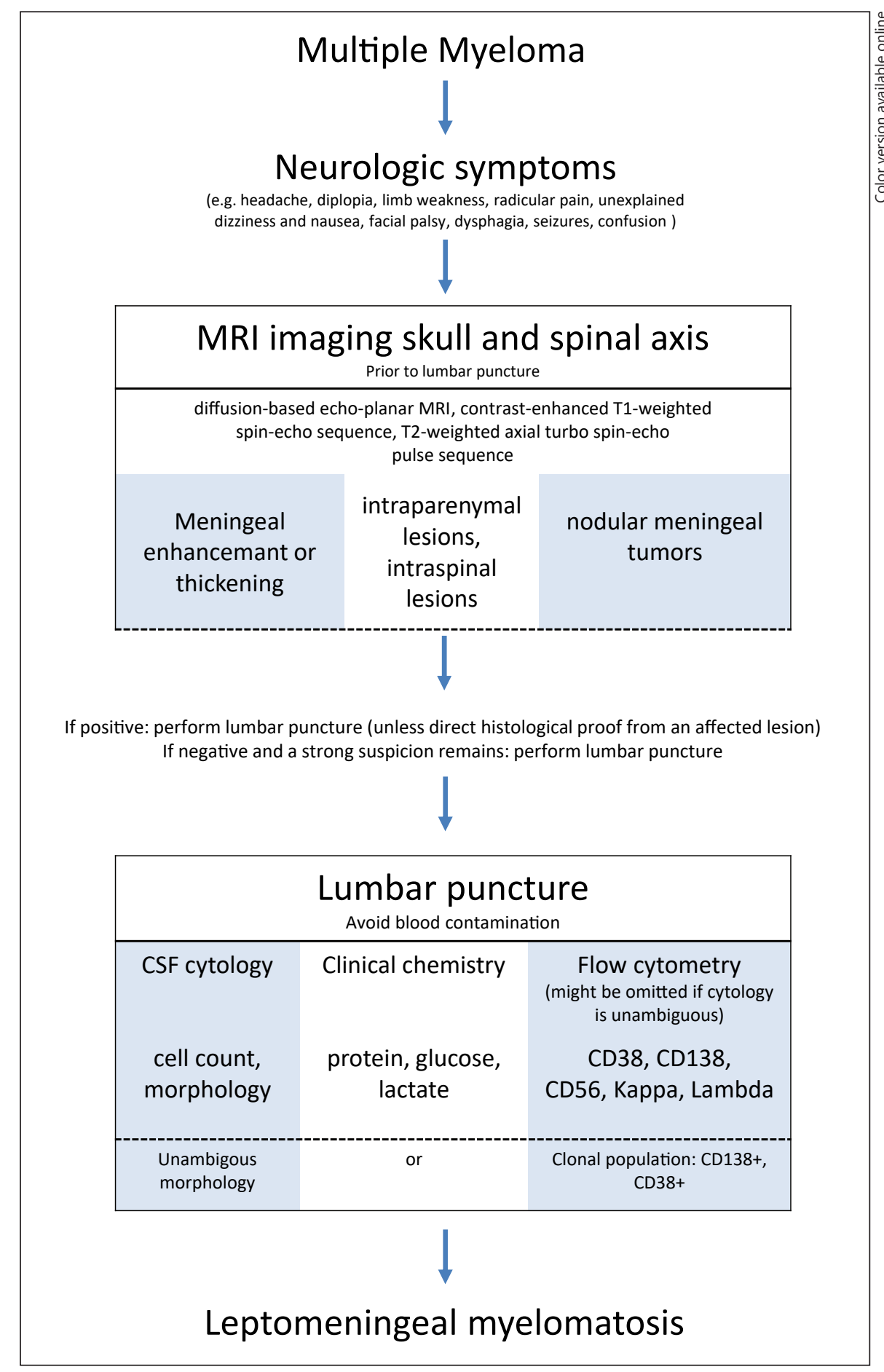

revealed intraparenchymal lesions (26\%) or dural disease (35\%). Imaging of the skull and spine are required for a complete workup.

Cytological analysis of CSF is the gold standard for the diagnosis of neoplastic meningitis. In patients with a low
CSF cell count or doubtful morphology, the addition of flow cytometry is very useful to discriminate reactive from malignant cells and to identify LMM rapidly [14]. In our case study, flow cytometry was able to add diagnostic reliability despite the low cell count (in patient Nos. 2, 3, 9, and 
10). Leptomeningeal disease underlies most malignancies of unfavorable prognosis. Despite CNS-targeted treatment, such as the intrathecal application of liposomal ARA-C, methotrexate, or radiotherapy, the prognosis of LMM remains very poor with a median survival of 2 months.

Our data indicate that, in LMM, the median survival is also $<3$ months. This is in line with 2 larger series that recently reported on the outcome of patients with CNS involvement of MM [6,7]. Paludo et al. [6] found an incidence of for myelomatous involvement in $0.7 \%$ of a large cohort of $>4,000$ patients diagnosed and treated at the Mayo Clinic; in this group of 29 patients, the median overall survival was only 3.4 months. There was no specific therapeutic intervention identified that could positively influence the natural cause of the disease. In a multi-institutional study reported by Jurczyszyn et al. [7], the median overall survival for the entire group of 172 patients was 7 months from the onset of CNS involvement. However, when they included the presence of $>1$ cytogenetic abnormality detected by FISH and at least 1 prior therapy, the median survival of this group of patients was only 2 months. This dismal prognosis may have been caused by the fact that most of the drugs used in myeloma do not penetrate the blood-brain barrier if used for systemic treatment [15]. Newer drugs such as carfilzomib, marizomib, and pomalidomide may penetrate the blood-brain barrier and so may be used as therapeutic options in such situations [5, 16-18]. We observed 1 long-term survivor with LMM (patient No. 2), who was diagnosed with CNS involvement after allogeneic stem cell transplantation. He was treated with intrathecal liposomal ARA-C, CNS irradiation, and lenalidomide/dexamethasone (Table 1) as previously described elsewhere [19]. Nevertheless, he eventually died $>3$ years after diagnosis of LMM due to a systemic relapse. In most of the malignancies, except for acute lymphoblastic leukemia and Burkitt's lymphoma, spread to the CSF is a late event during the course of disease. Our data show that this is also true for patients with MM; the median time for the diagnosis of LMM after the initial diagnosis is $>2$ years. Novel drugs, such as bortezomib, lenalidomide, or thalidomide allow long-term control of myeloma. If this also accounts for a higher rate of extramedullary disease presentations, including CNS myeloma is a matter for discussion and further investigation [20]. Therapeutic strategies, including drugs with a higher ability to penetrate the blood-brain barrier such as pomalidomide or newer proteasome inhibitors such as carfilzomib, may improve the prognosis.

From a biological point of view, however, it will eventually be important to target all subclones of the disease, if not up front then at least at the time of relapse or progression. This was demonstrated in 1 patient for whom we were able to perform whole-genome analysis of the disease at the same time point at different sites of presentation. Whereas the dominant CSF clone was characterized by hyperdiploidy like the clone at first presentation, the clone in the BM had lost this characteristic. Apart from this, copy number alterations, like gains at $8 \mathrm{q}$, were detected only in the CSF sample. This clonal evolution at different sites, particularly between BM and extramedullary disease might partially explain why patients may respond to treatment at one location but not at another. In a landmark paper, Keats et al. [21] were able to analyze the genomic landscape of a high-risk myeloma patient at 7 different time points, showing a dramatic linear evolution. This is a common phenomenon, recently demonstrated by a large systematic study of 51 patients in whom $>75 \%$ showed spatial genomic heterogeneity [22].

\section{Conclusion}

LMM occurs as a rare complication late in the course of MM. Up to now, no reliable clinical or cytogenetic parameters have been available to predict LMM occurrence. Known incidences may increase during the next decade, possibly due to more efficient systemic treatment options for advanced myeloma. A diagnosis can be made using a combination of MRI and CSF cytology and flow cytometry (Fig. 5). Even though no standard treatment for this clinical situation has been described or established, the potential therapeutic measures include combinations of radiotherapy, intrathecal treatment using methotrexate and liposomal ARA-C, and systemic treatment. Further studies to evaluate the effect of new drugs such as pomalidomide, carfilzomib, and marizomib on CNS disease in MM are warranted. Ultimately, the novel technical possibilities in detecting even subclonal disease might help to identify and tailor more effective treatment modalities.

\section{Acknowledgements}

This work was funded in part by the Deutsche Forschungsgemeinschaft (DFG) (Emmy-Noether program Kr3886/2-1 to J.K., KFO-216 to C.L.).

\section{Disclosure Statement}

The authors declare no conflicts of interest. 


\section{References}

1 Schluterman KO, Fassas AB, Van Hemert RL, Harik SI: Multiple myeloma invasion of the central nervous system. Arch Neurol 2004;61: 1423-1429.

2 Fassas AB, Muwalla F, Berryman T, Benramdane R, Joseph L, Anaissie E, et al: Myeloma of the central nervous system: association with high-risk chromosomal abnormalities, plasmablastic morphology and extramedullary manifestations. Br J Haematol 2002;117: 103-108.

3 Fassas AB, Ward S, Muwalla F, Van Hemert R, Schluterman K, Harik S, Tricot G: Myeloma of the central nervous system: strong association with unfavorable chromosomal abnormalities and other high-risk disease features. Leuk Lymphoma 2004;45:291-300.

4 Nieuwenhuizen L, Biesma DH: Central nervous system myelomatosis: review of the literature. Eur J Haematol 2008;80:1-9.

5 Yellu MR, Engel JM, Ghose A, Onitilo AA: Overview of recent trends in diagnosis and management of leptomeningeal multiple myeloma. Hematol Oncol 2016;34:2-8.

6 Paludo J, Painuly U, Kumar S, Gonsalves WI, Rajkumar V, Buadi F, et al: Myelomatous involvement of the central nervous system. Clin Lymphoma Myeloma Leuk 2016;16:644-654.

7 Jurczyszyn A, Grzasko N, Gozzetti A, Czepiel J, Cerase A, Hungria V, et al: Central nervous system involvement by multiple myeloma: a multi-institutional retrospective study of 172 patients in daily clinical practice. Am J Hematol 2016;91:575-580.

8 Chamberlain MC, Glantz M, Groves MD, Wilson WH: Diagnostic tools for neoplastic meningitis: detecting disease, identifying patient risk, and determining benefit of treatment. Semin Oncol 2009;36:S35-S45.
9 Quijano S, Lopez A, Manuel Sancho J, Panizo C, Deben G, Castilla C, et al: Identification of leptomeningeal disease in aggressive B-cell non-Hodgkin's lymphoma: improved sensitivity of flow cytometry. J Clin Oncol 2009;27: 1462-1469.

10 Hegde U, Filie A, Little RF, Janik JE, Grant N, Steinberg SM, et al: High incidence of occult leptomeningeal disease detected by flow cytometry in newly diagnosed aggressive B-cell lymphomas at risk for central nervous system involvement: the role of flow cytometry versus cytology. Blood 2005;105:496-502.

11 Bommer M, Nagy A, Schopflin C, Pauls S, Ringhoffer M, Schmid M: Cerebrospinal fluid pleocytosis: pitfalls and benefits of combined analysis using cytomorphology and flow cytometry. Cancer Cytopathol 2011;119:20-26.

12 Bommer $M$, von Harsdorf $S$, Dohner $H$, Bunjes D, Ringhoffer M: Neoplastic meningitis in patients with acute myeloid leukemia scheduled for allogeneic hematopoietic stem cell transplantation. Haematologica 2010;95: 1969-1972.

13 Pauls S, Fischer AC, Brambs HJ, Fetscher S, Hoche W, Bommer M: Use of magnetic resonance imaging to detect neoplastic meningitis: limited use in leukemia and lymphoma but convincing results in solid tumors. Eur J Radiol 2012;81:974-978.

14 Marini A, Carulli G, Lari T, Buda G, Lambelet $\mathrm{P}$, Ciancia EM, et al: Myelomatous meningitis evaluated by multiparameter flow cytometry: report of a case and review of the literature. J Clin Exp Hematop 2014;54:129-136.
15 Gozzetti A, Cerase A: Novel agents in CNS myeloma treatment. Cent Nerv Syst Agents Med Chem 2014;14:23-27.

16 Mussetti A, Dalto S, Montefusco V: Effective treatment of pomalidomide in central nervous system myelomatosis. Leuk Lymphoma 2013;54:864-866.

17 Gertz MA: Pomalidomide and myeloma meningitis. Leuk Lymphoma 2013;54:681682.

18 Abdallah AO, Atrash S, Shahid Z, Jameel M, Grazziutti M, Apewokin S, et al: Patterns of central nervous system involvement in relapsed and refractory multiple myeloma. Clin Lymphoma Myeloma Leuk 2014;14:211-214.

19 Chen CI, Masih-Khan E, Jiang H, Rabea A, Cserti-Gazdewich C, Jimenez-Zepeda VH, et al: Central nervous system involvement with multiple myeloma: long-term survival can be achieved with radiation, intrathecal chemotherapy, and immunomodulatory agents. $\mathrm{Br} \mathrm{J}$ Haematol 2013;162:483-488.

20 Varga C, Xie W, Laubach J, Ghobrial IM, O’Donnell EK, Weinstock M, et al: Development of extramedullary myeloma in the era of novel agents: no evidence of increased risk with lenalidomide-bortezomib combinations. Br J Haematol 2015;169:843-850.

21 Keats JJ, Chesi M, Egan JB, Garbitt VM, Palmer SE, Braggio E, et al: Clonal competition with alternating dominance in multiple myeloma. Blood 2012;120:1067-1076.

22 Rasche L, Chavan SS, Stephens OW, Patel PH, Tytarenko R, Ashby C, et al: Spatial genomic heterogeneity in multiple myeloma revealed by multi-region sequencing. Nat Commun 2017;8:268. 